\title{
Preoperative quality of life and surgical outcomes in gynecologic oncology patients: A new predictor of operative risk?
}

\author{
KM Doll ${ }^{1,2}$, AC Snavely ${ }^{3}$, A Kalinowski ${ }^{4}$, DE Irwin ${ }^{5}$, JT Bensen ${ }^{3}$, V Bae-Jump ${ }^{1,3}$, JF \\ Boggess $^{1,3}$, JT Soper ${ }^{1,3}$, WR Brewster ${ }^{1,3}$, and PA Gehrig ${ }^{1,3}$ \\ ${ }^{1}$ Division of Gynecologic Oncology, University of North Carolina School of Medicine, Chapel Hill, \\ NC \\ ${ }^{2}$ Cancer Care Quality Training Program, Division of Health Policy and Management, Gillings \\ School of Global Public Health, University of North Carolina, Chapel Hill, NC \\ ${ }^{3}$ Lineberger Comprehensive Cancer Center, University of North Carolina, Chapel Hill, NC \\ ${ }^{4}$ Department of Obstetrics and Gynecology, University of North Carolina School of Medicine, \\ Chapel Hill, NC \\ ${ }^{5}$ Department of Epidemiology, Gillings School of Global Public Health, University of North \\ Carolina, Chapel Hill, NC
}

\begin{abstract}
Objective-Quality of life (QoL) for women with gynecologic malignancies is predictive of chemotherapy related toxicity and overall survival but has not been studied in relation to surgical outcomes and hospital readmissions. Our goal was to evaluate the association between baseline, pre-operative QoL measures and 30-day post-operative morbidity and health resource utilization by gynecologic oncology patients.
\end{abstract}

Methods-We analyzed prospectively collected survey data from an institution-wide cohort study. Patients were enrolled from 8/2012 - 6/2013 and medical records data was abstracted (demographics, comorbid conditions, and operative outcomes). Responses from several validated health-related QoL instruments were collected. Bivariate tests and multivariable linear and logistic regression models were used to evaluate factors associated with QoL scores.

Results-Of 182 women with suspected gynecologic malignancies, $152(84 \%)$ were surveyed pre-operatively and $148(81 \%)$ underwent surgery. Uterine $(94 ; 63.5 \%)$, ovarian $(26 ; 17.5 \%)$,

(C) 2014 Elsevier Inc. All rights reserved.

Corresponding Author: Kemi M. Doll, MD, Division of Gynecologic Oncology, University of North Carolina - Chapel Hill, Physicians Office Building, 101 Manning Drive, Campus Box 7572, Chapel Hill, NC 27599, kmdoll@med.unc.edu, P: 919-966-5996, F: 919-843-5387.

We have no conflicts of interest to report.

Publisher's Disclaimer: This is a PDF file of an unedited manuscript that has been accepted for publication. As a service to our customers we are providing this early version of the manuscript. The manuscript will undergo copyediting, typesetting, and review of the resulting proof before it is published in its final citable form. Please note that during the production process errors may be discovered which could affect the content, and all legal disclaimers that apply to the journal pertain. 
cervical $(15 ; 10 \%)$, vulvar/vaginal $(8 ; 5.4 \%)$, and other $(5 ; 3.4 \%)$ cancers were represented. There were 37 (25\%) cases of postoperative morbidity (PM), 18 (12\%) unplanned ER visits, 9(6\%) unplanned clinic visits, and 17 (11.5\%) hospital readmissions(HR) within 30 days of surgery. On adjusted analysis, lower functional well-being scores resulted in increased odds of PM (OR 1.07, 95\% CI 1.01-.1.21) and HR (OR 1.11, 95\%CI 1.03-1.19). A subjective global assessment score was also strongly associated with HR (OR 1.89, 95\%CI 1.14, 3.16).

Conclusion-Lower pre-operative QoL scores are significantly associated with post-operative morbidity and hospital readmission in gynecologic cancer patients. This relationship may be a novel indicator of operative risk.

\section{Keywords}

Quality of life; Surgical complications; Postoperative recovery

\section{Introduction}

The primary treatment modality for many gynecologic malignancies is surgery, often followed by adjuvant chemotherapy and/or radiation. Surgery is often radical, with perioperative complication rates of up to $50 \%$ depending on cancer site (1-4). In addition, gynecologic oncology patients are frequently surgically and medically complex, which compounds surgical risk. This contributes to rates of post-operative morbidity between $20-30 \%$ and rates of hospital readmission of $10-15 \%$ after primary surgical management (5, $6)$.

Both postoperative morbidity and hospital readmission in cancer patients prolong surgical recovery, delay vital adjuvant treatment, increase overall health care costs and can have a negative psychosocial impact on the patient and her family $(7,8)$. The factors that contribute to surgical outcomes are multifactorial. Efforts to identify discrete predictive factors, including frailty measures, have largely focused on medical comorbidity and patient characteristics as assessed and interpreted by health care providers (9-11). Data on the relationship between patient-reported outcomes - information provided directly from the patient, without interpretation or modification - and surgical outcomes is limited.

Quality of life (QoL) assessments are a form of patient-reported outcomes, and have been validated in assessing disease burden, treatment, and prognosis across a spectrum of cancer sites (12-14). These scores are derived from QoL surveys, which are designed to measure physical, functional, social and/or emotional well-being domains. Within gynecologic oncology, specifically in women with ovarian cancer, QoL scores are predictive of disease status, chemotherapy toxicity, and overall survival(12,13, 15). The strongest associations have been found within physical and functional domains. In two large Gynecologic Oncology Group (GOG) chemotherapy trials, women in the lowest quartile of physical wellbeing scores had decreased overall survival $(12,15)$. In colon cancer patients undergoing surgery, preoperative patient-reported measures of poor functional status have been associated with postoperative morbidity and mortality, with lower scores being associated with increased risk (16-18). Such baseline factors that are found to predict poor surgical 
outcomes would represent new targets for intervention to improve the quality of surgical recovery, avoid delays in adjuvant therapy, and decrease cancer care costs.

Our primary study objective was to explore the association between preoperative, baseline QoL domain scores and postoperative morbidity and hospital readmission in gynecologic oncology patients. We hypothesized that worse QoL scores would be associated with poor surgical outcomes and this relationship would be strongest within the functional and physical wellbeing domains.

\section{Methods}

\section{Study Design, Enrollment, and Data Collection}

We conducted an analysis of data prospectively collected for a large hospital-based observational cohort. The Health Registry/Cancer Survivorship Cohort (HR/CSC) is an institutional review board approved University of North Carolina (UNC) Health Care registry of cancer patients that integrates a comprehensive database of clinical, epidemiological, and interview data, with repositories of biologic specimens and tumor tissue. Patients are identified and recruited through UNC Health Care oncology outpatient clinics with the following eligibility criteria: age 18 years or older; North Carolina mailing address; and English or Spanish proficiency. Patients who are unable to provide informed consent or participate in interview questionnaires are excluded. For this analysis, eligibility was further restricted to HR/CSC patients recruited through the gynecologic oncology clinics, who completed the baseline interview prior to any cancer treatments, and had documented 30 day post-operative follow up.

Interviews were conducted within 2 weeks of enrollment by trained staff using a computerassisted telephone interview software tool specifically developed for the HR/CSC. Interview questionnaire topics include medical and social histories, and general and cancer-specific health assessments. The following structured and validated questionnaires were included in the analysis: Functional Assessment of Cancer Therapy - General Population (FACT-GP), NIH Patient Reported Outcomes Measurement Information System (PROMIS@) global mental (GMH) and physical health (GPH), a modified Work Ability Index, PG-SGA (Patient Generated - Subjective Global Assessment), cancer-specific FACTs (Endometrial En, Ovarian - O, Vaginal/Vulvar - V, Cervical - Cx). The FACT-GP version 4 is a 21-item scale that measures health related QoL using four subscales: physical (PWB), functional (FWB), emotional (EWB), and social (SWB) wellbeing. The cancer-specific FACT scales include the FACT-GP in addition to multi-item subscales that measure cancer site-specific symptoms(19-22). The PROMIS@ v1.0 global is a 10-item scale that measures the domains of fatigue, physical function, pain, emotional distress, and social health(23). The modified work ability index includes a subset of questions from the original scale, designed to assess work ability compared to lifetime best, in relation to mental and physical demands, and sick leave(24). The specific question analyzed for this study was, "Assume that your ability to work at its best has a value of 10 points and 0 means that you cannot currently work at all. How many points would you give your current ability to work?" with a $0-10$ scale response. The PG-SGA is a measure of overall functional status on a 5 tier scale, mirroring 
the Eastern Cooperative Oncology Group (ECOG) performance status ratings(25), but from the patient's perspective(26).

Patient age, self-reported race/ethnicity, and employment status were abstracted from the HR/CSC baseline interview. The electronic medical record was reviewed (physician, nursing, and case management staff documentation) to abstract clinical data at the time of new patient visit (BMI, co-morbid conditions, mental health history, cancer site) and during the 30 day post-operative follow up window (surgical approach, intra/post-operative complications, discharge location, unplanned clinic or emergency room visits, readmission, and adjuvant treatment. Insurance status, at the time of new patient visit, was also abstracted from the medical record. All medical record data was limited to encounters at our institution. The medical record data file was then merged with the HR/CSC demographic and QoL data, using an honest broker model. The HR/CSC subsequently provided a de-identified data set for analysis.

\section{Statistical Analysis}

Summary statistics were generated using simple frequencies for categorical variables and mean/medians for continuous variables. Composite variables of major medical comorbidity, mental health diagnoses, and post-operative morbidity were created. The major comorbidity variable included notation in the record for at least one of these conditions: diabetes, pulmonary disease (chronic obstructive pulmonary disease, restrictive lung disease, home oxygen requirement), cardiac disease (congestive heart failure, history of MI, coronary artery disease), immunocompromised states (HIV, chronic steroid use), and chronic kidney disease. For the composite mental health variable, we combined any notated diagnosis of anxiety, depression, and chronic pain. Surgical morbidity was divided into intraoperative complications (bladder, ureteral, vascular, nerve, and GI injury) and post-operative complications (thromboembolic events, abscesses, cardiac event, renal insufficiency, pulmonary events, organ injury, wound infection, wound opening, ileus, blood transfusion, urinary tract infection). QoL scores were analyzed continuously and by 5 -point increments in relation to two outcomes: post-operative morbidity and hospital readmission (both 30 day post-operative measures). The 5-point increment was chosen due to the minimally important difference in most scale measures of 2-3 points (18-21). QoL scores were also treated as the outcome and compared between patients who did and did not experience postoperative morbidity and/or hospital readmission (analysis groups). Due to the modification of the work ability index, no summary score could be generated and analyzed, therefore a simple comparison of median responses to the selected question was performed between the analysis groups. Only employed patients had recorded responses for this question. Baseline characteristics between analysis groups were compared using Fisher's exact tests for categorical variables, and Student's t-tests for continuous variables. The relationship between the various QoL domains and post-operative outcomes were evaluated using univariable and multivariable linear and logistic regression models.

We included factors in the multivariable analysis if they were identified clinically and known to directly contribute to postoperative outcomes and if they differed substantially between the analysis groups. Established risk factors considered to impact post-operative 
morbidity were age, BMI, minimally invasive surgical approach, and major medical comorbidity (composite variable). The two factors considered to impact 30-day readmission were insurance status and vulvar surgery. Insurance status was considered because uninsured patients may require admission to access services that insured patients could receive as outpatients, thus increasing their risk of readmission. Vulvar surgery was considered because vulvar procedures have a significantly higher risk of late wound complications compared to abdominal surgery (1), and this could result in higher readmission rates compared to the other cancer sites. Using linear regression, multivariable adjusted means were calculated, and using logistic regression odds ratios were calculated and a graph of predicted probabilities was produced.

\section{Results}

Of 182 gynecologic oncology patients enrolled in the HR/CSC, there were 152 who met inclusion criteria and formed the study cohort. Of the 30 excluded, 27 did not complete baseline interviews (refusal $(n=20)$, contacted after surgery $(n=6)$, incomplete interview $(n=1))$ and 3 had surgery performed at outside facilities). Of the study cohort, 148/152 underwent surgical treatment and 4 received primary chemotherapy or radiation. The 4 patients treated with primary chemotherapy and/or radiation had advanced cervical $(n=2)$, $\operatorname{vulvar}(\mathrm{n}=1)$, and unspecified gynecologic $(\mathrm{n}=1)$ cancer and were excluded from the surgical outcomes analysis. There were no patients with dual primary cancer sites. Median age was 58.9 (range $28-90$, SD 13.0). Most patients were obese (BMI 230). The cohort had a median BMI of 33.5 (range 18 - 62, SD 8.7). There was substantial racial, economic, and social diversity (Table 1). There were 44 patients (29.0\%) with no medical comorbidity, 65 $(42.7 \%)$ with a non-major medical comorbidity, and 43 (28.3\%) with major medical comorbidity (as defined by the composite variable). With regard to mental health, 39 patients (25.7\%) had diagnoses of depression, anxiety, or chronic pain. All gynecologic cancer sites and stages were represented, in addition to 3 patients with suspected ovarian cancer, who underwent standard debulking surgery, with final pathology resulting with gastrointestinal primary malignancy (Table 1). Given that their surgical procedure was identical to the ovarian cancer patients, we included them in the analysis.

A range of surgical procedures was performed, with a fairly even distribution between minimally invasive and traditional open surgery (Table 2, Supplementary Table 1). Postoperative morbidity was common, with $25 \%$ of patients experiencing major and/or minor complications. Most patients with a complication experienced $\geq 1$ event (Table 2, Supplementary Table 2). Intensive care unit use, discharge to a subacute or acute rehabilitation facility, and death, were rare (Table 2). After outpatient procedure or discharge from an inpatient procedure, $33(22.3 \%)$ of patients required unanticipated health care resources defined as unplanned clinic or emergency room visits. There was only 1 patient with an emergency room visit who was not subsequently admitted to the hospital. Following primary surgical management, 94 (63.5\%) of patients were assigned to receive adjuvant therapy, of whom 74 (78.8\%) had documented treatment dates. The reasons for the absence of documented dates of treatment were: loss to follow up ( $\mathrm{N}=4)$; transfer of care 
with no further documentation $(\mathrm{N}=4)$; patient preference against treatment $(\mathrm{N}=9)$; treatment delay secondary to post-operative complications $(\mathrm{N}=2)$ and unknown $(\mathrm{N}=1)$.

\section{Univariable Analyses}

Several preoperative QoL scores were different between patients who did and did not experience postoperative morbidity and/or hospital readmission. In all cases of difference, mean QoL scores of the postoperative morbidity and hospital readmission groups were lower, indicating worse QoL (Table 3). Mean total FACT-GP scores differed between patients who experienced post-operative morbidity and those who did not (74.7 vs 81.5 , $\mathrm{p}=0.05)$. This was largely driven by the differences in the mean subscale of functional wellbeing ( 15.3 vs $18.7, \mathrm{p}=.01$ ). With examination of hospital readmission groups, the overall FACT-GP mean scores also differed, driven by the differing physical (17.2 vs 22.3, $\mathrm{p}=.003)$ and functional (12.8 vs $18.5, \mathrm{p}=.002)$ wellbeing subscales. Mean cancer-specific FACT-En, FACT-Cx, and FACT-V scores also differed with lower scores in the postoperative morbidity and readmission groups, with the largest group - Uterine cancer patients under the FACT-En -- reaching statistical significance (113.3 vs 140.7, p=.001) for association with readmission. The FACT-Ovarian score relationship was reversed with regard to postoperative morbidity (high scores in the postoperative morbidity group). The readmission relationship was consistent with the other survey instruments. Readmitted patients had lower mean PROMIS global mental and physical health scores. In comparing the responses from the overall work ability question, from the modified Work Ability Index, the median response for the readmission group (scale 0 -10) was 4.7 (IQR 1.5 - 8.0) compared to a median response of 8.4 (IQR $7.6-9$ ) in the group that was not readmitted $(\mathrm{p}=0.002)$.

\section{Adjusted (Multivariable) analyses}

Of the identified potential adjustment factors, only surgical route differed significantly by post-operative morbidity group. There were $26 / 37$ laparotomies $(70.3 \%)$ in the postoperative morbidity group, and 44/67 (39.6\%) in the group without post-operative morbidity. Age, BMI, medical comorbidity, mental health diagnoses, and surgical history were similar between the groups. Table 4 shows the risk odds ratio from the multivariable logistic regression model, adjusting for surgical route, for each 1-point decrease in score. The FWB score ranges from $0-25$. Based on this model each 5-point increase corresponds to a $27 \%$ decrease in the odds of postoperative morbidity. Given the relationship between mental health diagnoses and quality of life, we also performed an adjusted analysis for anxiety, depression, and chronic pain. With this adjustment, post-operative morbidity patients still had mean FWB scores 3.25 points lower than patients without postoperative morbidity ( $\mathrm{p}=0.02)$. There were no other domain scores that remained significantly associated with postoperative morbidity when adjusted for surgical route or anxiety/ depression/chronic pain.

For the 30 day readmission analysis, neither of the identified adjustment factors (insurance status, vulvar surgery) differed statistically; however, the distributions were not balanced. For insurance status, there were no Medicare/Medicaid patients in the readmitted group compared to 12 in the group without readmission. With regard to vulvar surgery, there were 
$3 / 17(17.7 \%)$ in the readmission group and 5/131 (3.8\%) in the group without readmission. Therefore, both variables were considered as confounders. Age, BMI, medical comorbidity, and surgical route did not differ between the readmission groups. Given the number of readmission events $(\mathrm{N}=17)$, we could only account for a single covariate in the model, which was adjusting for insurance type. The FACT-GP, FWB, PWB, PROMIS global physical health, and PG-SGA scores were all significantly associated with 30-day readmission (Table 4). The strongest association among the subscales was with FWB. Each 1 point increase in FWB score, resulted in a 10\% decrease in the odds of 30 day readmission. Evaluated in 5-point increments, this corresponded to a $40 \%$ decrease in the odds. Each one unit increase in PG-SGA assessment was associated with 1.89 times the odds of readmission (OR 1.89, 95\%CI $1.14-3.16, \mathrm{p}=0.012$ ). In this scale, increasing score represents decreasing functional ability. Endometrial cancer patients were the largest cancersite subset, and the corresponding FACT-En was also associated with 30 day readmission (OR 1.04, 95\%CI 1.01 - 1.06). We performed the same set of analyses, adjusting for vulvar surgery and again for bowel procedures, with similar results (not shown). Finally, we adjusted for mental health diagnoses, and the trend in mean QoL scores remained the same, with lower scores associated with 30 day readmission (data not shown). The predicted probabilities of 30-day readmission by FWB subscale are shown in Figure 1.

\section{Discussion}

In an observational cohort study of gynecologic oncology patients, our analysis demonstrates that baseline, preoperative QoL scores are associated with postoperative surgical outcomes and hospital readmission. Lower scores in physical and functional domains are associated with increased risk of postoperative morbidity and 30 day readmission.

It is the goal of all cancer surgeons to both proceed with a successful operation and minimize perioperative complications that lead to prolonged recovery and may delay subsequent adjuvant cancer therapy. The quality of surgical recovery is equally as important as the quality of the surgical operation. In cancer patients, the quality surgical recovery has lasting effects. Surgery is frequently the first step in multi-modality treatment with the goal of curing the cancer. It follows, then, that the relative ease (quality) with which a patient recovers can impact his or her physical, functional, and emotional resilience for adjuvant therapies to come. Even for those who do not require adjuvant therapy, post-operative complications can have long lasting physical, social, emotional and financial implications for the patient and her family.

QoL domain scores are patient-reported assessments of physical, functional, and emotional resilience. These scores are known to be predictive of treatment toxicity and survival in ovarian cancer patients undergoing chemotherapy $(12,15)$. Within the clinical trial GOG 172, patients with the lowest quartile of physical wellbeing domain scores were more likely to die (OR 1.81, 95\% CI 1.2-2.7) and for each mean point increase in this score, there was a $20 \%$ decrease in death rate. In our study of ovarian, endometrial, cervical, and vulvar cancer patients, we have found that for every 5-point increase in functional wellbeing score, there is a $27 \%$ decrease in the odds of postoperative morbidity and a $40 \%$ decrease in the odds of 
readmission. Among several different assessment tools we analyzed, we show that this relationship was most consistent among measurements of physical and functional wellbeing. This consistency implies that our patients' self-assessed physical and functional ability are important contributing factors to the quality of their surgical recovery.

Our findings have several implications. Patient reported measures may be just as important as physician assessments of surgical risk. This additional information on a patient's global status could help improve informed consent and shared decision making for risky surgical procedures, especially in the emotional context of a new cancer diagnosis. For physicians, improving the accuracy of surgical risk assessment would also aid greatly in patient selection for surgery. Finally, in the same way we view medical risk factors, poor nutritional status, and polypharmacy (27-29), these physical and functional deficits can be targets for intervention. Occupational and physical therapy are designed to improve functional and physical status and their use for surgical cancer patients in the pre-operative and/or immediate postoperative setting, to our knowledge, has not be explored.

Our study is strengthened by its observational design, capturing a racially and economically diverse patient population. As these patients were not defined by enrollment into a clinical trial, we feel these results can be translated to a wider population not captured in cooperative group trials. Our cohort also had a complication and readmission rate comparable to recently published studies of gynecologic oncology populations, furthering the generalizability(1-6).

Our results represent preliminary evidence and must be verified with additional, larger prospective studies. We were limited by the degree of documentation in the medical record, although we reviewed physician, nursing, and ancillary staff notes. Our study involved multiple cancer sites within gynecologic oncology and a variety of surgical procedures, introducing many potential confounders. Our sample size limited our ability to create complex multivariable models and our results for cancer-site specific instruments for cervical, ovarian, and vulvar cancer are very limited by the small number of outcomes within each group. However, we could account for the multiple cancer sites and routes of surgery partially, by evaluating both the largest subgroup (endometrial cancer) and by removing the highest risk site (vulvar cancer). With both of these sensitivity analyses, the QoL score relationship remained significant and consistent with findings among other populations $(18,23,24)$. We note also that these QoL measures were gathered in the context of a larger cohort study, with a baseline hour-long interview incorporating other patientreported tools. This format would not be appropriate for general clinical care. Given the versatility of survey administration (in person, telephone, written), and the increasing ability to communicate with patients electronically, we can conceive of a web-based platform of evaluation where patients could directly enter information and reports could be generated for the surgeon, alongside radiological and pathology data.

Improved surgical risk assessment improves patient counseling and informed consent, enhances surgeon decision making through improved patient selection, and provides novel targets for pre- and post-operative intervention. In cancer patients, this additional information could lend weight to crucial decisions between primary medical or surgical management. We are currently conducting a prospective observational study, administering 
QoL assessments preoperatively, and at 1,3 and 6 months postoperatively to verify these results and explore the short and long-term quality of life trends after surgery. If verified, this will mean that it is possible to identify a subset of surgical patients, who may be at high risk for morbidity, and therefore candidates for pre or post-operative functional intervention. This is an important first step in a patient-centered approach to improve the quality of surgical recovery in cancer care.

\section{Supplementary Material}

Refer to Web version on PubMed Central for supplementary material.

\section{Acknowledgments}

Research reported in this publication was supported by the National Cancer Institute of the National Institutes of Health under Award Number R25CA116339. The content is solely the responsibility of the authors and does not necessarily represent the official views of the National Institutes of Health.

The authors thank the HR/CSC participants for their important contributions. The UNC Health Registry/Cancer Survivorship Cohort is funded in part by the UNC Lineberger Comprehensive Cancer Center's University Cancer Research Fund. This project was reviewed and approved by the Human Research Protections Program (IRB Number: 09-0605) at the University of North Carolina at Chapel Hill.

\section{References}

1. Dittmer C, Fischer D, Diedrich K, Thill M. Diagnosis and treatment options of vulvar cancer: a review. Archives of gynecology and obstetrics. 2012; 285(1):183-93. [PubMed: 21909752]

2. Wright JD, Lewin SN, Deutsch I, Burke WM, Sun X, Neugut AI, et al. Defining the limits of radical cytoreductive surgery for ovarian cancer. Gynecologic oncology. 2011; 123(3):467-73. [PubMed: 21958535]

3. Boggess JF, Gehrig PA, Cantrell L, Shafer A, Ridgway M, Skinner EN, et al. A comparative study of 3 surgical methods for hysterectomy with staging for endometrial cancer: robotic assistance, laparoscopy, laparotomy. American journal of obstetrics and gynecology. 2008; 199(4):360 e1-9. [PubMed: 18928974]

4. Wright JD, Herzog TJ, Neugut AI, Burke WM, Lu YS, Lewin SN, et al. Comparative effectiveness of minimally invasive and abdominal radical hysterectomy for cervical cancer. Gynecologic oncology. 2012; 127(1):11-7. [PubMed: 22735788]

5. Clark RM, Growdon WB, Wiechert A, Boruta D, Del Carmen M, Goodman AK, et al. Patient, treatment and discharge factors associated with hospital readmission within 30 days after surgical cytoreduction for epithelial ovarian carcinoma. Gynecologic oncology. 2013; 130(3):407-10. [PubMed: 23747329]

6. Henretta MS, Scalici JM, Engelhard CL, Duska LR. The revolving door: hospital readmissions of gynecologic oncology patients. Gynecol Oncol. 2011; 122(3):479-83. Epub 2011/06/15. [PubMed: 21664659]

7. Wright JD, Herzog TJ, Neugut AI, Burke WM, Lu YS, Lewin SN, et al. Effect of radical cytoreductive surgery on omission and delay of chemotherapy for advanced-stage ovarian cancer. Obstet Gynecol. 2012; 120(4):871-81. [PubMed: 22996105]

8. Jencks SF, Williams MV, Coleman EA. Rehospitalizations among patients in the Medicare fee-forservice program. New England Journal of Medicine. 2009; 360(14):1418-28. [PubMed: 19339721]

9. Revenig LM, Canter DJ, Taylor MD, Tai C, Sweeney JF, Sarmiento JM, et al. Too frail for surgery? Initial results of a large multidisciplinary prospective study examining preoperative variables predictive of poor surgical outcomes. Journal of the American College of Surgeons. 2013; 217(4): 665-70 e1. [PubMed: 24054409]

10. Davenport DL, Bowe EA, Henderson WG, Khuri SF, Mentzer RM Jr. National Surgical Quality Improvement Program (NSQIP) risk factors can be used to validate American Society of 
Anesthesiologists Physical Status Classification (ASA PS) levels. Annals of surgery. 2006; 243(5): 636-41. discussion 41-4. [PubMed: 16632998]

11. Reisinger KW, van Vugt JL, Tegels JJ, Snijders C, Hulsewe KW, Hoofwijk AG, et al. Functional Compromise Reflected by Sarcopenia, Frailty, and Nutritional Depletion Predicts Adverse Postoperative Outcome After Colorectal Cancer Surgery. Annals of surgery. 2014 Epub 2014/03/22.

12. von Gruenigen VE, Huang HQ, Gil KM, Frasure HE, Armstrong DK, Wenzel LB. The association between quality of life domains and overall survival in ovarian cancer patients during adjuvant chemotherapy: a Gynecologic Oncology Group Study. Gynecol Oncol. 2012; 124(3):379-82. [PubMed: 22119995]

13. Wenzel L, Huang HQ, Monk BJ, Rose PG, Cella D. Quality-of-life comparisons in a randomized trial of interval secondary cytoreduction in advanced ovarian carcinoma: a Gynecologic Oncology Group study. Journal of Clinical Oncology. 2005; 23(24):5605-12. [PubMed: 16110020]

14. Quinten C, Coens C, Mauer M, Comte S, Sprangers MA, Cleeland C, et al. Baseline quality of life as a prognostic indicator of survival: a meta-analysis of individual patient data from EORTC clinical trials. The lancet oncology. 2009; 10(9):865-71. [PubMed: 19695956]

15. Wenzel LB, Huang HQ, Armstrong DK, Walker JL, Cella D, Gynecologic Oncology G. Healthrelated quality of life during and after intraperitoneal versus intravenous chemotherapy for optimally debulked ovarian cancer: a Gynecologic Oncology Group Study. Journal of Clinical Oncology. 2007; 25(4):437-43. [PubMed: 17264340]

16. Anthony T, Hynan LS, Rosen D, Kim L, Nwariaku F, Jones C, et al. The association of pretreatment health-related quality of life with surgical complications for patients undergoing open surgical resection for colorectal cancer. Ann Surg. 2003; 238(5):690-6. Epub 2003/10/28. [PubMed: 14578731]

17. Saxton A, Velanovich V. Preoperative frailty and quality of life as predictors of postoperative complications. Ann Surg. 2011; 253(6):1223-9. Epub 2011/03/18. [PubMed: 21412145]

18. Ihemelandu CU, McQuellon R, Shen P, Stewart JH, Votanopoulos K, Levine EA. Predicting postoperative morbidity following cytoreductive surgery with hyperthermic intraperitoneal chemotherapy (CS+HIPEC) with preoperative FACT-C (Functional Assessment of Cancer Therapy) and patient-rated performance status. Ann Surg Oncol. 2013; 20(11):3519-26. Epub 2013/06/12. [PubMed: 23748607]

19. Cella DF, Tulsky DS, Gray G, Sarafian B, Linn E, Bonomi A, et al. The Functional Assessment of Cancer Therapy scale: development and validation of the general measure. J Clin Oncol. 1993; 11(3):570-9. Epub 1993/03/01. [PubMed: 8445433]

20. Cella D. FACT-En (Version 4). 1997

21. Basen-Engquist K, Bodurka-Bevers D, Fitzgerald MA, Webster K, Cella D, Hu S, et al. Reliability and validity of the functional assessment of cancer therapy-ovarian. J Clin Oncol. 2001; 19(6): 1809-17. Epub 2001/03/17. [PubMed: 11251013]

22. Janda M, Obermair A, Cella D, Crandon AJ, Trimmel M. Vulvar cancer patients' quality of life: a qualitative assessment. Int J Gynecol Cancer. 2004; 14(5):875-81. Epub 2004/09/14. [PubMed: 15361198]

23. Hays RD, Bjorner JB, Revicki DA, Spritzer KL, Cella D. Development of physical and mental health summary scores from the patient-reported outcomes measurement information system (PROMIS) global items. Qual Life Res. 2009; 18(7):873-80. Epub 2009/06/23. [PubMed: 19543809]

24. Ilmarinen J. The Work Ability Index. Occupational Medicine. 2007; 57(2):160.

25. Oken MM, Creech RH, Tormey DC, Horton J, Davis TE, McFadden ET, et al. Toxicity and response criteria of the Eastern Cooperative Oncology Group. Am J Clin Oncol. 1982; 5(6):649_ 55. Epub 1982/12/01. [PubMed: 7165009]

26. Ottery FD. Definition of standardized nutritional assessment and interventional pathways in oncology. Nutrition. 1996; 12(1 Suppl):S15-9. Epub 1996/01/01. [PubMed: 8850213]

27. Englesbe MJ, Terjimanian MN, Lee JS, Sheetz KH, Harbaugh CM, Hussain A, et al. Morphometric age and surgical risk. J Am Coll Surg. 2013; 216(5):976-85. Epub 2013/03/26. [PubMed: 23522786] 
28. Lee JS, He K, Harbaugh CM, Schaubel DE, Sonnenday CJ, Wang SC, et al. Frailty, core muscle size, and mortality in patients undergoing open abdominal aortic aneurysm repair. J Vasc Surg. 2011; 53(4):912-7. Epub 2011/01/11. [PubMed: 21215580]

29. Badgwell B, Stanley J, Chang GJ, Katz MH, Lin HY, Ning J, et al. Comprehensive geriatric assessment of risk factors associated with adverse outcomes and resource utilization in cancer patients undergoing abdominal surgery. J Surg Oncol. 2013; 108(3):182-6. Epub 2013/06/28. [PubMed: 23804149] 


\section{Research Highlights}

1. Patient-reported pre-operative $\mathrm{QoL}$ measures are associated with postoperative outcomes in gynecologic oncology patients.

2. Measures of lower functional and physical ability are most strongly correlated with risk of post-operative morbidity and hospital readmissions.

3. Identifying QoL deficits pre-operatively can improve patient selection and counseling, and targeting of high-risk patients for pre- and post-operative intervention. 


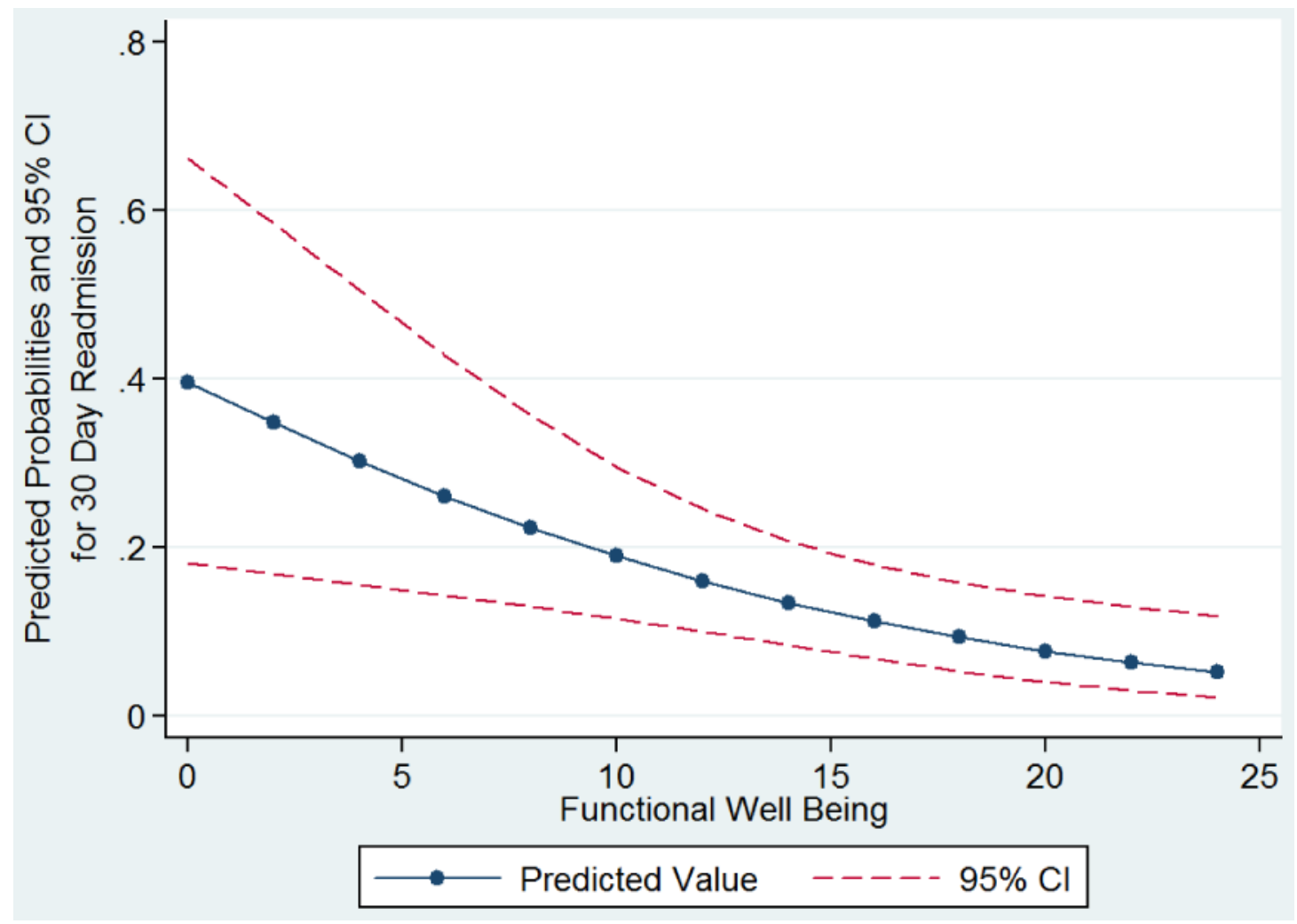

Figure 1. Predicted probabilities for 30 Day Readmission by Pre-operative Functional Well Being Score 
Table 1

Overall Study Cohort Characteristics

\begin{tabular}{|c|c|c|c|}
\hline Variable & & $\mathbf{N}$ & $\%$ \\
\hline \multirow[t]{4}{*}{ Age } & $18-30$ years & 3 & 2.0 \\
\hline & $31-50$ years & 30 & 19.7 \\
\hline & $51-70$ years & 92 & 60.5 \\
\hline & Over 70 years & 27 & 17.8 \\
\hline \multicolumn{4}{|l|}{ BMI } \\
\hline & Under 25.0 & 27 & 17.8 \\
\hline & $25.0-29.9$ & 27 & 17.8 \\
\hline & $30.0-39.9$ & 68 & 44.7 \\
\hline & Over 40.0 & 30 & 19.7 \\
\hline \multicolumn{4}{|c|}{ Race/Ethnicity 1} \\
\hline & White & 120 & 79.0 \\
\hline & Black & 22 & 14.5 \\
\hline & Hispanic & 3 & 2 \\
\hline & Native American & 3 & 2 \\
\hline & Asian & 2 & 1.3 \\
\hline & Other & 2 & 1.3 \\
\hline \multicolumn{4}{|l|}{ Insurance } \\
\hline & Private & 108 & 71.0 \\
\hline & Uninsured & 32 & 21.0 \\
\hline & Medicare only & 7 & 4.6 \\
\hline & Medicaid & 5 & 3.2 \\
\hline \multicolumn{4}{|c|}{ Employment } \\
\hline & Part Time & 24 & 15.8 \\
\hline & Full Time & 51 & 33.6 \\
\hline & Unemployed/Retired & 77 & 50.1 \\
\hline \multicolumn{4}{|c|}{ Marital Status } \\
\hline & Married/Partnered & 67 & 44.0 \\
\hline & Single/Divorced/Widowed & 17 & 11.2 \\
\hline & Unknown & 68 & 44.5 \\
\hline \multicolumn{4}{|c|}{ Past Medical History } \\
\hline & Obesity & 98 & 64.5 \\
\hline & Prior Laparotomy & 35 & 23.0 \\
\hline & Diabetes $^{2}$ & 30 & 19.7 \\
\hline & Pulmonary Disease ${ }^{2}$ & 4 & 2.6 \\
\hline & HTN & 75 & 49.3 \\
\hline & Chronic Kidney Disease ${ }^{2}$ & 2 & 1.3 \\
\hline & Cardiac Disease (CHF/CAD/MI) ${ }^{2}$ & 15 & 9.9 \\
\hline & Arthritis & 13 & 8.6 \\
\hline
\end{tabular}




\begin{tabular}{|c|c|c|c|}
\hline Variable & & $\mathbf{N}$ & $\%$ \\
\hline & Immunocompromise $^{2}$ & 2 & 1.3 \\
\hline & History of Cancer & 28 & 18.4 \\
\hline & Substance Abuse & 18 & 11.8 \\
\hline & Major Comorbidity ${ }^{3}$ & 43 & 28.3 \\
\hline \multicolumn{4}{|l|}{ Mental Health } \\
\hline & Substance Abuse & 18 & 11.8 \\
\hline & Depression & 24 & 15.8 \\
\hline & Anxiety & 18 & 11.8 \\
\hline & Chronic Pain & 5 & 3.3 \\
\hline \multicolumn{4}{|l|}{ Cancer Site } \\
\hline & Uterine & 94 & 61.8 \\
\hline & Ovarian & 26 & 17.1 \\
\hline & Cervical & 17 & 11.1 \\
\hline & Vulva/Vaginal & 9 & 5.9 \\
\hline & Gyn $\operatorname{NOS}^{4}$ & 3 & 2.0 \\
\hline & $\mathrm{GI}^{5}$ & 3 & 2.0 \\
\hline \multicolumn{4}{|l|}{ Cancer Stage } \\
\hline & Stage I - II & 98 & 64.5 \\
\hline & Stage III - IV & 46 & 30.3 \\
\hline & Unstaged & 8 & 5.3 \\
\hline
\end{tabular}

${ }^{1}$ NIH defined self-reported race/ethnicity.

2 Indicates inclusion in Major Comorbidity composite variable.

3 Indicates inclusion in Major Comorbidity composite variable.

${ }^{4}$ Gyn Not Otherwise Specified - Either gynecologic cancer of unknown origin (2) or dual gynecologic primary(1).

${ }^{5}$ Gastrointestinal -3 patients with suspected ovarian cancer that had primary GI malignancy on final pathology. 
Table 2

Overall Study Cohort Treatment Outcomes

\begin{tabular}{|c|c|c|c|}
\hline Variable & & $\mathbf{N}$ & $\%$ \\
\hline \multicolumn{4}{|c|}{ Primary Treatment } \\
\hline & Surgery & 148 & 97.4 \\
\hline & Chemo/Rads 1 & 4 & 2.6 \\
\hline \multicolumn{4}{|c|}{ Surgical Approach } \\
\hline & MIS $^{2}$ & 78 & 52.7 \\
\hline & Laparotomy & 62 & 41.9 \\
\hline & Groin & 8 & 5.4 \\
\hline \multicolumn{4}{|c|}{ Postoperative Course (30 Day) } \\
\hline & Intra op complication ${ }^{3}$ & 14 & 9.5 \\
\hline & Postop morbidity 4 & 37 & 25.0 \\
\hline & ICU Admission & 6 & 4.0 \\
\hline & Death & 1 & 0.7 \\
\hline \multicolumn{4}{|c|}{ Discharge Location } \\
\hline & Home & 143 & 96.6 \\
\hline & $\mathrm{SNF}^{3}$ & 4 & 2.7 \\
\hline & Acute Rehab ${ }^{6}$ & 1 & 0.7 \\
\hline \multicolumn{4}{|c|}{ Unplanned Post-op Resource Use (30 Day) } \\
\hline & Clinic Visit & 9 & 6.1 \\
\hline & ER Visit & 18 & 12.1 \\
\hline & Readmission & 17 & 11.5 \\
\hline \multicolumn{4}{|c|}{ Adjuvant Treatment } \\
\hline & Recommended & 94 & 63.5 \\
\hline & Not Recommended & 54 & 36.5 \\
\hline & Treatment Started & 74 & $78.8 \%$ \\
\hline & No Treatment started & 20 & $21.2 \%$ \\
\hline
\end{tabular}

${ }^{1}$ Chemotherapy and/or Radiation

2 Minimally invasive surgery including laparoscopic, robotic, or hysteroscopic approach

3 Includes bladder, ureteral, vascular, nerve, and GI injury

4 Includes VTE/PE, Abscesses, Cardiac event, renal insufficiency, pulmonary events, organ injury, wound infection, wound opening, ileus, blood transfusion, urinary tract infection

${ }^{5}$ Skilled nursing facility or Sub-acute rehabilitation facility

${ }^{6}$ Acute inpatient rehabilitation facility 


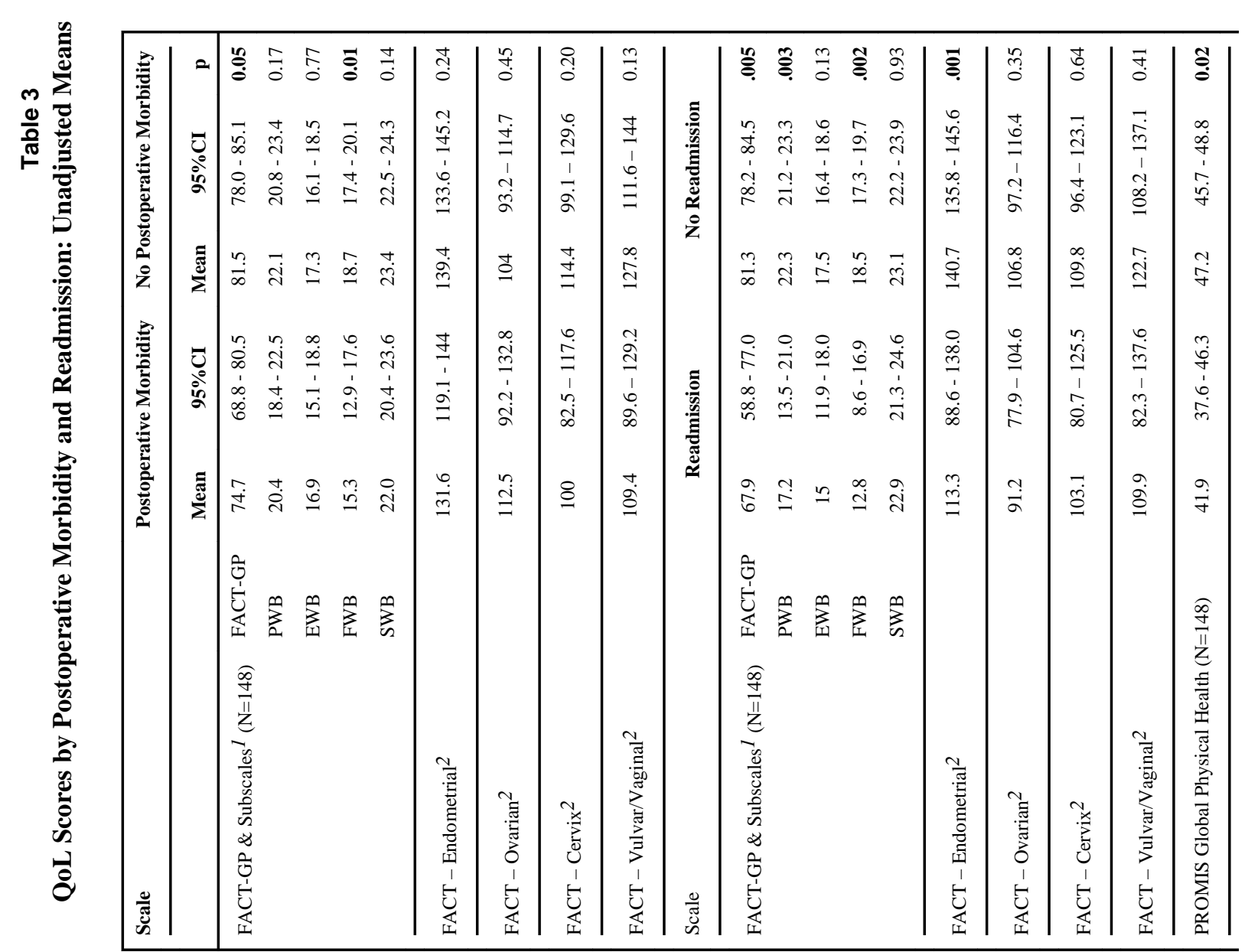




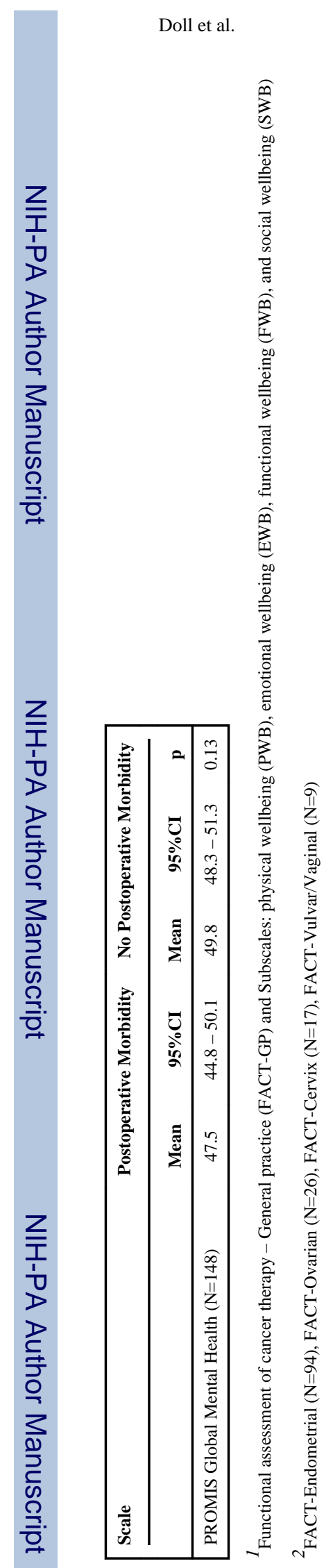

Page 18 
Table 4

Odds Ratio of Surgical Outcomes by Preoperative Quality of Life Assessment

\begin{tabular}{|lccc|}
\hline & Odds Ratio & p-value & 95\% Confidence Interval \\
\hline \multicolumn{4}{c}{ Postoperative Morbidity } \\
\hline FWB & 1.07 & 0.01 & $1.01-1.21$ \\
\hline \multicolumn{4}{c}{ Hospital Readmission ${ }^{2}$} \\
\hline FACT-GP & 1.03 & 0.03 & $1.01,1.06$ \\
FWB & 1.11 & 0.006 & $1.03,1.19$ \\
PWB & 1.10 & 0.008 & $1.02,1.18$ \\
PROMIS Global & 1.07 & 0.04 & $1.01,1.05$ \\
Physical Health FACT-En & \\
\end{tabular}

Odds ratios are reported for a 1-point decrease for each scale. Lower scores are associated with increased odds of postoperative morbidity and/or hospital readmission.

\footnotetext{
${ }^{1}$ Adjusted for surgical route

2 Adjusted for insurance status

3 Endometrial cancer patients only, $\mathrm{N}=86$
} 